Cite this: Phys. Chem. Chem. Phys., 2012, 14, 3737-3740

\title{
On the structure of the $\mathrm{Au}_{18}(\mathrm{SR})_{14}$ cluster $\dagger$
}

\author{
Alfredo Tlahuice and Ignacio L. Garzón* \\ Received 15th December 2011, Accepted 26th January 2012 \\ DOI: $10.1039 / \mathrm{c} 2 \mathrm{cp} 24016 \mathrm{e}$
}

First principles calculations are used for a systematic search of the lowest-energy (most-stable) structure of the recently synthesized $\mathrm{Au}_{18}(\mathrm{SR})_{14}$ cluster. A comparison of the calculated optical absorption and electronic circular dichroism spectra, which are highly sensitive to the cluster structure and chirality, with the experimental spectra of the glutathione-protected gold cluster, $\mathrm{Au}_{18}(\mathrm{SG})_{14}$, is used to discriminate between low-energy isomers of the $\mathrm{Au}_{18}(\mathrm{SR})_{14}\left(\mathrm{R}=\mathrm{CH}_{3}\right)$ cluster. From the good agreement between calculated and measured spectra, it is predicted that the structure of the $\mathrm{Au}_{18}(\mathrm{SR})_{14}$ cluster consists of a prolate $\mathrm{Au}_{8}$ core covered with two dimer (SR-Au-SR-Au-SR) and two trimer (SR-Au-SR-Au-SR-Au-SR) motifs. These results provide additional evidence on the existence of longer trimer motifs as protecting units of small thiolated gold clusters.

Studies on ligand-protected metal clusters became an important field of research due to their interesting properties and promising applications in nanotechnology. ${ }^{1}$ Despite the tremendous progress achieved during the past fifteen years on the synthesis and physicochemical characterization of small $(<2 \mathrm{~nm})$ thiolateprotected gold clusters, several issues of scientific and technological interest still remain unknown. ${ }^{1}$ One of the challenges is to develop and improve the synthetic chemistry for synthesizing a series of size-discrete $\mathrm{Au}_{n}(\mathrm{SR})_{m}$ clusters with high purity and high yield, followed by their crystallization and X-ray total structure determination, ${ }^{1}$ as was the case for $(n, m)=(25,18)$, $(38,24)$, and $(102,44) .^{2-4}$ A precise knowledge of the cluster atomic structure is a fundamental step toward a full understanding of other physicochemical properties of thiolated gold clusters. $^{1-4}$

Gold clusters covered by glutathione, $\mathrm{Au}_{n}(\mathrm{SG})_{m}$, and other related ligands had been synthesized and size-separated by Tsukuda's group. ${ }^{5,6}$ High mass resolution and accurate mass calibration in their electrospray ion mass spectrometry (ESI-MS) data allowed the exact determination of the cluster chemical composition. ${ }^{5,6}$ The nine smallest compounds were assigned to

Instituto de Física, Universidad Nacional Autónoma de México, Apartado Postal 20-364, 01000 México D. F., México.

E-mail: tlahuicef@fisica.unam.mx,garzon@fisica.unam.mx

$\dagger$ Electronic supplementary information (ESI) available: Methodology to find the initial cluster structures; calculated optical absorption spectra of the $\mathrm{Au}_{12}(\mathrm{SR})_{9}{ }^{+}$and $\mathrm{Au}_{20}(\mathrm{SR})_{16}$ clusters; structures of the $\mathrm{Au}_{8}$ cores, simulated XRD patterns, and $x y z$ relaxed coordinates of Iso1-Iso4. Kohn-Sham diagram levels and tables with the excitation energies, oscillator strengths, atomic orbital contributions, and weights of the electronic transitions. See DOI: 10.1039/c2cp24016e cluster sizes with $n: m$ ratio: $10: 10,15: 13,18: 14,22: 16$, $22: 17,25: 18,29: 20,33: 22$, and $39: 24$, respectively. ${ }^{5,6}$ Interestingly, the most abundant compound with enhanced stability against unimolecular decomposition was found to be the $\mathrm{Au}_{18}(\mathrm{SG})_{14}$ cluster, ${ }^{5}$ although it also had been shown that the $25: 18$ clusters exhibit the highest stability against core etching. ${ }^{7}$

More recently, Dass' group has synthesized a mixture of thiolated gold clusters, $\mathrm{Au}_{n}(\mathrm{RS})_{m}\left(\mathrm{R}=\mathrm{CH}_{2} \mathrm{CH}_{2} \mathrm{Ph}\right)$, in the $\mathrm{Au}_{16}-\mathrm{Au}_{31}$ size range using a surfactant-free synthesis method. ${ }^{8}$ Again, the most abundant cluster size in the mixture, detected by matrix-assisted laser desorption time of flight mass spectrometry (MALDI-TOF-MS), corresponded to the $\mathrm{Au}_{18}(\mathrm{SR})_{14}$ cluster. $^{8}$ It is expected that the peculiar stability and higher relative abundance of the $18: 14$ cluster should motivate its crystallization and further X-ray total structure determination; however, no such studies have been reported so far.

On the theoretical side, there have not been calculations on the physicochemical properties of the $\mathrm{Au}_{18}(\mathrm{SR})_{14}$ cluster, although Jiang and collaborators speculated that its structure may be described as an $\mathrm{Au}_{8}$ core protected by two $\mathrm{V}$-shaped (RS-Au-SR-Au-SR) dimer motifs and two U-shaped (RS-Au-SR-Au-SR-Au-SR) trimer motifs. ${ }^{9}$ This suggestion was based on previous theoretical studies on the geometric structure of the $\mathrm{Au}_{12}(\mathrm{SR})_{9}{ }^{+10}$ and $\mathrm{Au}_{20}(\mathrm{SR})_{16}{ }^{9,11}\left(\mathrm{R}=\mathrm{CH}_{3}\right)$ clusters that had also been synthesized. ${ }^{12,13}$

In this communication, we present theoretical results that predict the geometrical structure of the $\mathrm{Au}_{18}(\mathrm{SR})_{14}\left(\mathrm{R}=\mathrm{CH}_{3}\right)$ cluster, which indicate that indeed it corresponds to an $\mathrm{Au}_{8}$ core (two fused tetrahedral $\mathrm{Au}_{4}$ units) covered by two dimer and two trimer motifs, in accordance with the "divide and protect" scheme. ${ }^{14}$ This structure assignment is supported by the good agreement obtained between the calculated optical absorption and electronic circular dichroism spectra and those measured on the $\mathrm{Au}_{18}(\mathrm{SG})_{14}$ samples. ${ }^{5,15}$ The present theoretically-calculated physicochemical properties of $\mathrm{Au}_{18}(\mathrm{SR})_{14}$ are timely and relevant not only because this cluster is specially peculiar due to its higher relative stability and abundance, $, 5,8$ but also because its crystallization and X-ray total structure determination would require a while. On the other hand, the predicted structure of the $\mathrm{Au}_{18}(\mathrm{SR})_{14}$ cluster, based on a prolate $\mathrm{Au}_{8}$ core covered by two dimer and two trimer motifs, provides additional evidence on the existence of the longer U-shaped trimer motifs as protecting units of small thiolated gold clusters, which were originally considered in structural studies of the $\mathrm{Au}_{20}(\mathrm{SR})_{16}{ }^{9,11}$ cluster and of the high energy isomers of $\mathrm{Au}_{38}(\mathrm{SR})_{24}{ }^{16}$ 
After a systematic search of lowest-energy isomers of small thiolated gold clusters: $\mathrm{Au}_{n}(\mathrm{SR})_{m}{ }^{+}(n=12-15, m=9-12)$ and $\mathrm{Au}_{n}(\mathrm{SR})_{m}(n=16-20, m=12-16)$; guided by previous theoretical studies on $\mathrm{Au}_{12}(\mathrm{SR})_{9}{ }^{+}$(see ref. 10) and $\mathrm{Au}_{20}(\mathrm{SR})_{16},{ }^{9,11}$ and following the staple fitness concept ${ }^{17}$ (see $\mathrm{ESI} \dagger$ section); a set of initial structures for the $\mathrm{Au}_{18}(\mathrm{SR})_{14}$ $\left(\mathrm{R}=\mathrm{CH}_{3}\right)$ cluster were optimized by density functional theory (DFT), within the generalized-gradient approximation (GGA). All DFT-GGA calculations were carried out using the PBE functional for the exchange and correlation (XC) terms, ${ }^{18}$ the LANL2DZ (19 valence electrons) basis set for $\mathrm{Au}$, and the (all-electron) 6-31G(d,p) basis set for S, C, and $\mathrm{H}$, as implemented in the Gaussian03 (G03) program package. ${ }^{19}$ The choice of the PBE functional is justified since it has been shown that other meta-GGA functionals, such as the nonempirical Tao-Perdew-Staroverov-Scuseria (TPSS) and the M06L one, provide similar trends in the relative energies of gold sulfide nanoclusters. ${ }^{20}$ Structural optimizations were performed without symmetry restrictions, using a force tolerance criterion of $0.01 \mathrm{eV}^{-1}$, followed by a cluster normal modes calculation. Time-dependent DFT (TD-DFT), as implemented in G03, was utilized for the study of the optical and chiroptical cluster properties, through the calculation of its excitation energies, and oscillator and rotatory strengths. ${ }^{19}$ To calculate the optical absorption and circular dichroism spectra, particularly, in the low energy region $(1.0-3.5 \mathrm{eV})$, the lowest 70 excited (singlet) states were considered, using the same $\mathrm{XC}$ functional and basis set as for the structural calculations. To test the reliability of the present TD-DFT methodology, a comparison between the calculated optical absorption spectra of the $\mathrm{Au}_{12}\left(\mathrm{SCH}_{3}\right)_{9}{ }^{+}$and $\mathrm{Au}_{20}(\mathrm{SR})_{16}$ clusters with previous calculations was performed, ${ }^{10,11}$ which indicates a good agreement, as shown in the ESI $\dagger$ section.

The most stable structures (lowest-energy minima, confirmed by a normal modes analysis) calculated for the $\mathrm{Au}_{18}(\mathrm{SR})_{14}$ cluster correspond to an $\mathrm{Au}_{8}$ core, based on two interconnected tetrahedral $\mathrm{Au}_{4}$ units, covered by two dimer and two trimer motifs. Three of them (Iso1-Iso3) are displayed in Fig. 1. Interestingly, a high-energy isomer (Iso4) was also obtained after the relaxation of a bicapped octahedron covered by two dimer and two trimer motifs. Table 1 shows
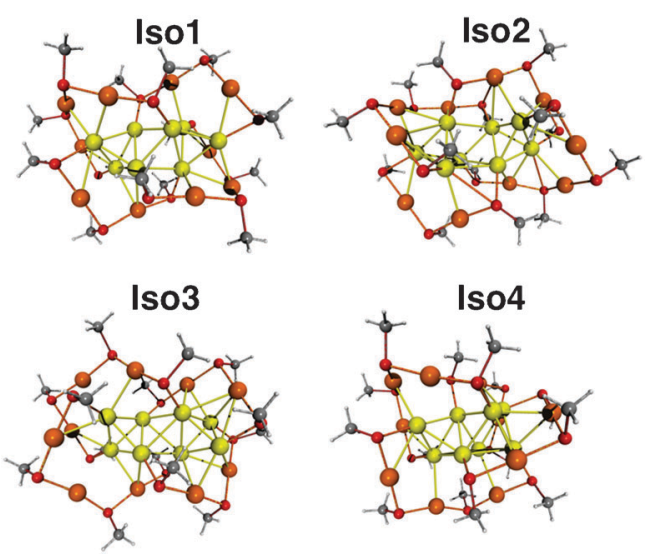

Fig. 1 Optimized structures of Iso1-Iso4. The S atoms are in red, $\mathrm{C}$ atoms are in gray, $\mathrm{H}$ atoms are in white. Au atoms in the dimer and trimer motifs are in orange and the core Au atoms are in yellow.
Table 1 Relative total energy and HOMO-LUMO (HL) gap of the Iso1-Iso4 isomers

\begin{tabular}{lll}
\hline Isomer & Relative total energy/eV & HL gap/eV \\
\hline Iso1 & 0.00 & 1.63 \\
Iso2 & 0.14 & 1.61 \\
Iso3 & 0.19 & 1.54 \\
Iso4 & 0.25 & 0.99 \\
\hline
\end{tabular}

the relative total energies of the four isomers, which indicate that indeed a higher energetic stability is obtained when two dimers and two trimer motifs protect a prolate $\mathrm{Au}_{8}$ core.

Bond length analysis of the optimized isomers confirms that the elongated $\mathrm{Au}_{8}$ core of Iso1-Iso3 can be considered as two fused $\mathrm{Au}_{4}$ tetrahedral units with distinct relative orientations (see Fig. S3, ESI $\dagger$ ) that depend on the way in which the dimer and trimer motifs are anchored on each $\mathrm{Au}_{4}$ unit. The average bond length between the $\mathrm{Au}_{4}$ units is longer than those within a single $\mathrm{Au}_{4}$ unit by about $0.25 \AA$. The major structural difference between the lowest-energy isomer, Iso1, and the next higher energy isomer, Iso2, is related to a distinct relative orientation of the $\mathrm{Au}_{4}$ units, which increases the number of $\mathrm{Au}-\mathrm{Au}$ bonds between them in the case of Iso1. The $\mathrm{Au}_{8}$ core of Iso3 shows a similar relative orientation of the $\mathrm{Au}_{4}$ units as Iso2, but a distinct arrangement of the protecting dimer and trimer motifs, with respect to those existing in Iso1 and Iso2. In Iso4, the longer U-shaped trimer motifs require to be anchored to distant $\mathrm{Au}$ atoms on the octahedral subunit and to the capping ones; however this optimized structure is $0.25 \mathrm{eV}$ higher in energy than that of Iso1. Relaxed atomic coordinates for all isomers are provided in the ESI $\dagger$ section.

Further structural analysis of the isolated $\mathrm{Au}_{8}$ cores shows that the one forming the lowest-energy isomer, Iso1, has the most distorted $\mathrm{Au}_{8}$ core, and only with a loose tolerance of $0.9 \AA$, it changes from $C_{1}$ to $C_{2}$ symmetry, while Iso2 with a small tolerance of $0.2 \AA$ acquires $C_{2}$ symmetry. Iso3 has $C_{\mathrm{s}}$ symmetry with a tolerance of $0.2 \AA$, and with a tolerance of $0.5 \AA$ shifts to a $D_{2 \mathrm{~d}}$ symmetry. Nevertheless, the calculated overall point symmetry of the entire cluster $\left(\mathrm{Au}_{8}\right.$ core plus the 2 dimer and 2 trimer motifs) is $C_{1}$. These results are in contrast with those obtained for the most stable isomers of the $\mathrm{Au}_{20}(\mathrm{SR})_{16}$ cluster, whose $\mathrm{Au}_{8}$ elongated cores, formed by two edge-fused tetrahedral $\mathrm{Au}_{4}$ units, protected by four trimer motifs, have near- $D_{2 \mathrm{~d}}$ symmetry. ${ }^{11}$ The $C_{1}$ symmetry in Iso1-Iso4 can be attributed to the different length of the $\mathrm{V}$-shaped dimer and the U-shaped trimer motifs that induces stronger core distortions when they mix together forming asymmetric patterns in the protecting layer of the most stable isomers of the $\mathrm{Au}_{18}(\mathrm{SR})_{14}$ cluster. The X-ray diffraction (XRD) curves of Iso1-Iso4 were also simulated and are shown in Fig. S4 (ESI $\dagger)$. Iso1-Iso3 with a similar prolate $\mathrm{Au}_{8}$ core exhibit a single strongest peak at $\sim 3.8 \mathrm{~nm}^{-1}$, similarly to the calculated data for the most stable isomers of the $\mathrm{Au}_{20}(\mathrm{SR})_{16}$ cluster. ${ }^{10}$ A more intense peak at $\sim 3.9 \mathrm{~nm}^{-1}$ and a qualitative distinct profile were obtained for the XRD pattern of Iso4, mainly due to its bicapped octahedron core.

The most stable isomers of $\mathrm{Au}_{18}(\mathrm{SR})_{14}$ are also distinguishable by their electronic properties. Fig. 2 and Fig. S5 (ESI $\dagger$ ) display the Kohn-Sham (KS) molecular orbital (MO) energy levels 


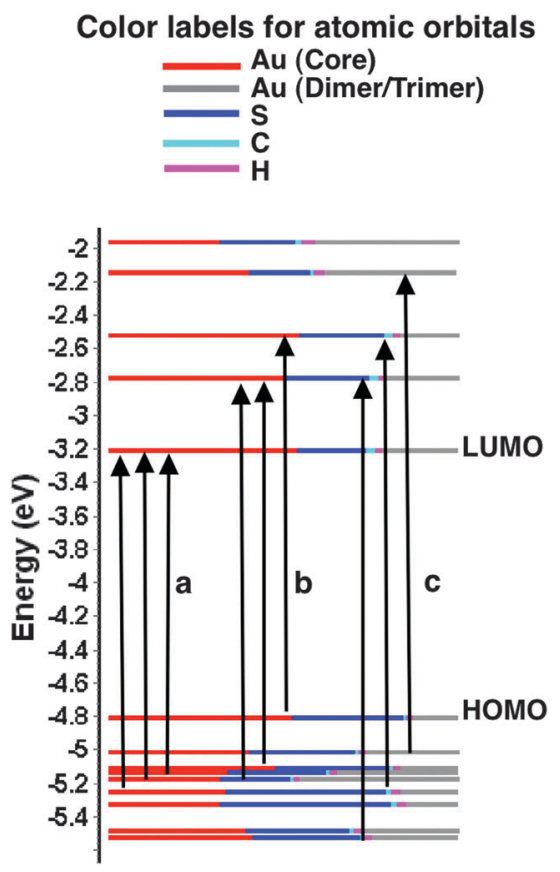

Fig. 2 Kohn-Sham molecular orbital energy levels diagram and atomic orbital components for isomer Iso2. Arrows show the electronic excitations contributing to the three peaks $\mathbf{a}, \mathbf{b}$, and $\mathbf{c}$ in the optical absorption spectra shown in Fig. 3.

diagram and the atomic orbital components in each $\mathrm{MO}$ for Iso1-Iso4, whereas Table 1 shows their different HOMO-LUMO (HL) gap values. It should be noted that Iso1-Iso3 with a prolate $\mathrm{Au}_{8}$ core protected with two dimer and two trimer motifs have smaller HL gaps (1.63-1.54 eV), compared with that calculated $(2.26 \mathrm{eV})$ for $\mathrm{Au}_{20}(\mathrm{SR})_{16}$ with a similar core, but protected with four trimer motifs. Despite this reduction in the HL gap, the most stable isomers of $\mathrm{Au}_{18}(\mathrm{SR})_{14}$ still should be considered as clusters with a relatively large HL gap, if it is recalled that the very stable $\left[\mathrm{Au}_{25}(\mathrm{SR})_{18}\right]^{-}$anionic cluster has an HL gap of $\sim 1.2 \mathrm{eV} .{ }^{14 b}$ In fact, the higher electronic stability of the prolate $\mathrm{Au}_{8}$ core structure may be understood on the basis of the nonspherical shell model, considering 4 delocalized valence electrons in the $\mathrm{Au}_{18}(\mathrm{SR})_{14}$ cluster, in close analogy to the $\mathrm{Au}_{20}(\mathrm{SR})_{16}$ case. $^{11}$

It is well known that the optical absorption spectrum is highly dependent on the cluster geometry, such that it can be used for the structure assignment of a thiolated gold cluster. ${ }^{3 b, 11}$ Fig. 3 (left column) shows a comparison between the calculated optical absorption spectra for Iso1-Iso4 and the experimental curve for $\mathrm{Au}_{18}(\mathrm{SG})_{14}$, which display characteristic peaks related to electronic transitions at 2.18, 2.54 and $2.80 \mathrm{eV} .^{5,15}$ The three more intense bands or characteristic peaks (labeled as $\mathbf{a}, \mathbf{b}$, and $\mathbf{c}$, and centered at the energy values given in parentheses) in the calculated spectra that are in a better agreement with those of the experimental curve correspond to Iso1. They are indicated as $\mathbf{a}(2.14 \mathrm{eV}), \mathbf{b}(2.54 \mathrm{eV})$, and $\mathbf{c}(2.87 \mathrm{eV})$ on the blue curve in Fig. 3. This agreement also includes the matching of the shoulder in the first peak and of the whole experimental profile with the theoretical lineshape obtained through the broadening of the associated oscillator strengths, represented as blue vertical bars. The calculated

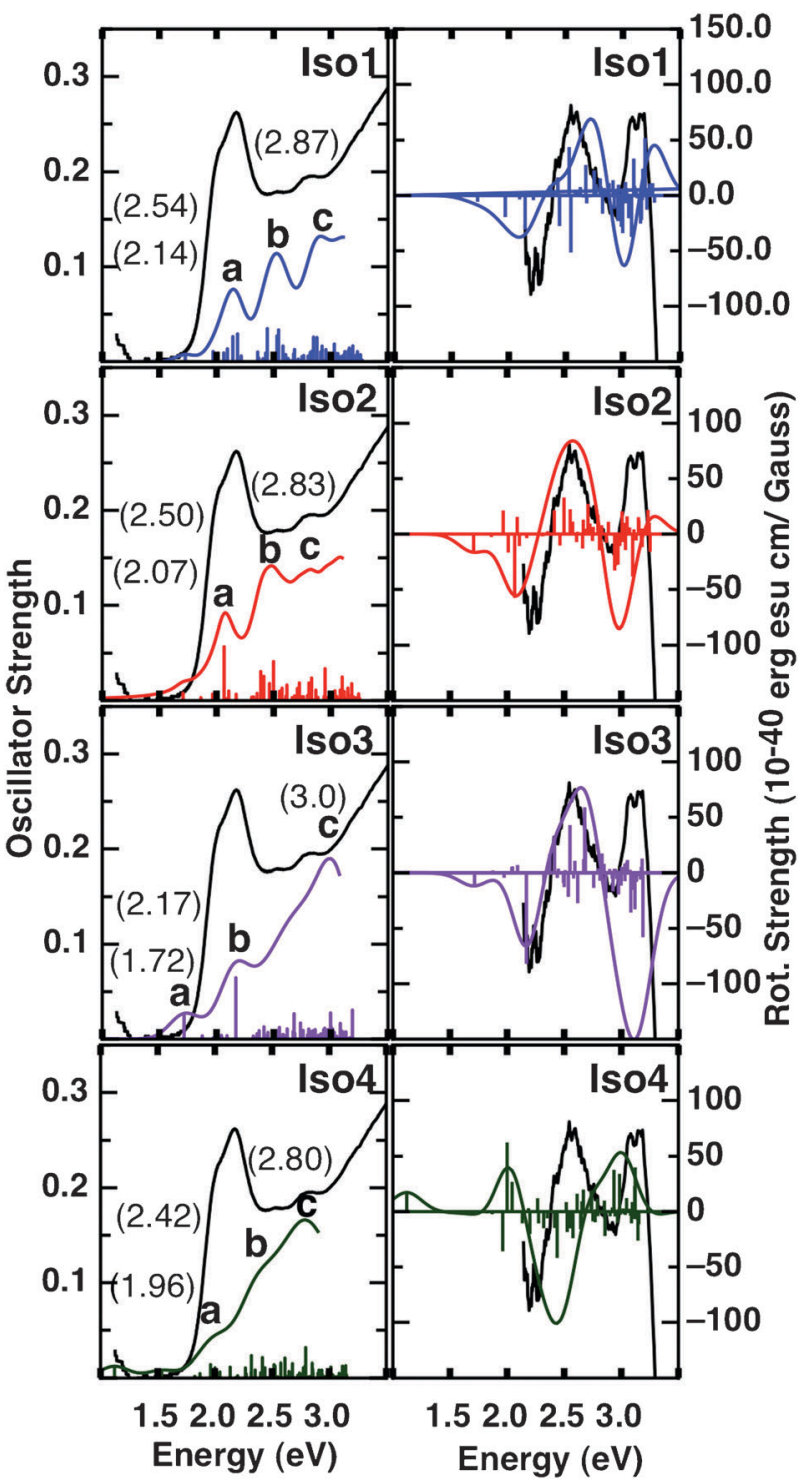

Fig. 3 Optical absorption and electronic circular dichroism spectra calculated for Iso1-Iso4, and their comparison with the experimental data (black curves). The experimental curves were taken from the data measured for $\mathrm{Au}_{18}(\mathrm{SG})_{14}$, reported in ref. 15. A Gaussian broadening of $0.1 \mathrm{eV}$ has been used.

lineshape of Iso2 could also be a candidate for a good matching with the experimental curve, however, the relative intensities of $\mathbf{b}$ and $\mathbf{c}$ peaks in the red curve are in less agreement with the corresponding relative intensities of the experimental curve. The calculated optical absorption spectra of Iso3 are yet in lesser agreement with the experimental data, also showing relative strong transitions at energies below $2 \mathrm{eV}$ that are not seen in the experimental curve. Since no agreement was obtained for the calculated optical absorption of Iso4, it is concluded that a bicapped octahedron core would not be forming the structure of the $\mathrm{Au}_{18}(\mathrm{SR})_{14}$ cluster.

The KS molecular orbital energy levels and their atomic orbital components of Iso2, depicted in Fig. 2, show that the contributions to the three strong absorption peaks $(\mathbf{a}-\mathbf{c})$ in the optical absorption are not only coming from the atoms in 
the $\mathrm{Au}_{8}$ core, but also from those forming the two dimer and two trimer motifs. In fact, according to Table S2 (ESI $\dagger$ ), the sets of occupied orbitals HOMO -8 to HOMO and unoccupied orbitals LUMO to LUMO + 3, which are involved in the electronic transitions giving rise to peaks $\mathbf{a}-\mathbf{c}$, are composed by a comparable proportion of orbitals from the core Au atoms (32-54\%), and those coming from the S (18-32\%) and $\mathrm{Au}$ (14-46\%) atoms forming the dimer and trimer motifs. This behavior was also found for Iso1 and Iso3, but it is qualitatively different from that obtained for the larger $\mathrm{Au}_{20}(\mathrm{SR})_{16}$ and $\left[\mathrm{Au}_{25}(\mathrm{SR})_{18}\right]^{-}$clusters, where the $\mathrm{Au}_{8}$ and $\mathrm{Au}_{13}$ cores, respectively, provide the main contribution to the low energy most intense peaks of the optical absorption spectrum..$^{1,21}$

Distinct circular dichroism (CD) calculated spectra for Iso1-Iso4 are displayed on the right column of Fig. 3, which indicate a strong sensitivity of the isomer chiral structures to the cluster optical activity. The calculated CD spectra of Iso1 and Iso2, with two alternating positive and negative peaks in the 1.5-3.5 eV range, show the best overall agreement with the experimental curve measured for the $\mathrm{Au}_{18}(\mathrm{SG})_{14}$ cluster, ${ }^{15}$ which exhibits similar intense peaks, except for the much weaker second negative one. This agreement together with the one obtained for the optical absorption spectra strongly suggest that the atomic configuration of most stable isomer, Iso1, is the best candidate structure for the $\mathrm{Au}_{18}(\mathrm{SR})_{14}$ cluster. Similarly to the optical absorption spectra, the electronic transitions giving rise to the most intense peaks in the CD spectra of Iso1-Iso4 are between molecular orbitals, composed by both core $\mathrm{Au}$ and dimer-trimer Au plus $\mathrm{S}$ atomic orbitals in comparable proportions. This behavior indicates that the origin of the optical activity in $\mathrm{Au}_{18}(\mathrm{SR})_{14}$ is related to the chirality of the whole ligand-protected cluster, instead of an intrinsic chirality of the $\mathrm{Au}_{8}$ core, or the formation of chiral patterns from the dimer or trimer motifs arrangement on an achiral $\mathrm{Au}_{8}$ core. This type of global cluster chirality would be a specific characteristic of smaller (molecular-like) ligandprotected clusters, as compared with other mechanisms that explain the optical activity found for larger $\left[\mathrm{Au}_{25}(\mathrm{SG})_{18}\right]^{-}$and $\mathrm{Au}_{38}(\mathrm{SR})_{24}$ chiral clusters. $^{22}$

In summary, we have calculated electronic, optical and chiroptical properties of a set of isomers (Iso1-Iso4) of the $\mathrm{Au}_{18}\left(\mathrm{SCH}_{3}\right)_{14}$ cluster, finding the most stable isomer, Iso1, as the one that better matches the experimental optical and chiroptical data of $\mathrm{Au}_{18}(\mathrm{SG})_{14}$. Although a good overall agreement was also found for Iso2, isomerisation at room temperature would be unlikely since the energy difference between both isomers is $0.14 \mathrm{eV}$. The predicted structure indicates that a prolate $\mathrm{Au}_{8}$ core can be protected not only by four trimer motifs as in the $\mathrm{Au}_{20}(\mathrm{SR})_{16}$ cluster, but also by a combination of two dimer and two trimer motifs. However, by examining the energetics of the $\mathrm{Au}_{18}(\mathrm{SR})_{14}+\frac{1}{2}(\mathrm{AuSR})_{4} \rightarrow$ $\mathrm{Au}_{20}(\mathrm{SR})_{16}$ reaction, where $(\mathrm{AuSR})_{4}$ is the well known cyclic tetramer, we obtained a stability higher by $0.45 \mathrm{eV}$ for the $\mathrm{Au}_{20}(\mathrm{SR})_{16}$ cluster. These results should motivate further synthesis, size separation, and physicochemical characterization of the chiral $18: 14$ thiolated gold clusters to confirm their relative higher abundance as well as the predicted structure, and the existence of the longer U-shaped (RS-Au-SR-Au-SR-Au-SR) trimer motifs, as protecting units of small thiolated gold clusters.

We thank professor Tatsuya Tsukuda for providing us the numerical data of the optical absorption and CD spectra of the $\mathrm{Au}_{18}(\mathrm{SG})_{14}$ cluster. We acknowledge support from Conacyt-México under Project 80610. Calculations were done using resources from the Supercomputing Center DGTIC-UNAM.

\section{Notes and references}

1 R. Jin, Nanoscale, 2010, 2, 343-362.

2 P. D. Jadzinnsky, G. Calero, C. J. Ackerson, D. A. Bushnell and R. D. Kornberg, Science, 2007, 318, 430-433.

3 (a) M. W. Heaven, A. Dass, P. S. White, K. M. Holt and R. W. Murray, J. Am. Chem. Soc., 2008, 130, 3754-3755; (b) M. Zhu, C. M. Aikens, F. J. Hollander, G. C. Schatz and R. Jin, J. Am. Chem. Soc., 2008, 130, 5883-5885.

4 H. Qian, W. T. Eckenhoff, Y. Zhu, T. Pintauer and R. Jin, J. Am. Chem. Soc., 2010, 132, 8280-8281.

5 Y. Negishi, K. Nobusada and T. Tsukuda, J. Am. Chem. Soc., 2005, 127, 5261-5270.

6 Y. Negishi, Y. Takasugi, S. Sato, H. Yao, K. Kimura and T. Tsukuda, J. Phys. Chem. B, 2006, 110, 12219.

7 Y. Shichibu, Y. Negishi, H. Tsunoyama, M. Kanehara, T. Teranishi and T. Tsukuda, Small, 2007, 3, 835-839.

8 S. M. Reilly, T. Krick and A. Dass, J. Phys. Chem. C, 2010, 114, 741-745.

9 D.-E. Jiang, W. Chen, R. L. Whetten and Z. Chen, J. Phys. Chem. C, 2009, 113, 16983-16987.

10 D.-E. Jiang, R. L. Whetten, W. Luo and S. Dai, J. Phys. Chem. C, 2009, 113, 17291-17295.

11 Y. Pei, Y. Gao, N. Shao and X. C. Zeng, J. Am. Chem. Soc., 2009, 131, 13619-13621.

12 Y. Zhang, S. M. Shuang, C. Dong, C. K. Lo, M. C. Paau and M. M. F. Choi, Anal. Chem., 2009, 81, 1676-1685.

13 M. Zhu, H. Qian and R. Jin, J. Am. Chem. Soc., 2009, 131, $7220-7221$.

14 (a) H. Häkkinen, M. Walter and H. Grönbeck, J. Phys. Chem. B, 2006, 110, 9927-9931; (b) J. Akola, M. Walter, R. L. Whetten, H. Häkkinen and H. Grönbeck, J. Am. Chem. Soc., 2008, 130, 3756-3757.

15 T. Tsukuda, H. Tsunoyama and Y. Negishi, in Metal Nanoclusters in Catalysis and Materials Science: The Issue of Size Control, ed. B. Corain, G. Schmid and M. Toshima, Elsevier, Amsterdam, 2008, p. 373.

16 I. L. Garzón, C. Rovira, K. Michaelian, M. R. Beltrán, J. Junquera, P. Ordejón, E. Artacho, D. Sánchez-Portal and J. M. Soler, Phys. Rev. Lett., 2000, 85, 5250-5251.

17 D.-E. Jiang, Chem.-Eur. J., 2011, 17, 12289-12293.

18 J. P. Perdew, K. Burke and M. Ernzerhof, Phys. Rev. Lett., 1996, 77, 3865-3868.

19 M. J. Frisch, et al., Gaussian 03, revision E.01, Gaussian, Inc, Wallingford, CT, 2004.

20 D.-E. Jiang, M. Walter and S. Dai, Chem.-Eur. J., 2010, 16, 4999-5003.

21 C. M. Aikens, J. Phys. Chem. Lett., 2011, 2, 99-104.

22 (a) A. Sánchez-Castillo, C. Noguez and I. L. Garzón, J. Am. Chem. Soc., 2010, 132, 1504-1505; (b) O. López-Acevedo, H. Tsunoyama, T. Tsukuda, H. Häkkinen and C. M. Aikens, J. Am. Chem. Soc., 2010, 132, 8210-8218; (c) M. Zhu, H. Qian, X. Meng, S. Jin, Z. Wu and R. Jin, Nano Lett., 2011, 11, 3963-3969; (d) H. Qian, M. Zhu, C. Gayathri, R. G. Gil and R. Jin, ACS Nano, 2011, 5, 8935-8942. 